Cahiers de Narratologie

Analyse et théorie narratives

$20 \mid 2011$

Voix off et narration cinématographique

\title{
Voix-off et film-fable : le cinéma d'éducation populaire à l'épreuve du parlant
}

\section{Pascal Laborderie}

\section{(2) OpenEdition}

Journals

Édition électronique

URL : http://journals.openedition.org/narratologie/6332

DOI : 10.4000/narratologie.6332

ISSN : 1765-307X

Éditeur

LIRCES

Référence électronique

Pascal Laborderie, «Voix-off et film-fable : le cinéma d'éducation populaire à l'épreuve du parlant », Cahiers de Narratologie [En ligne], 20 | 2011, mis en ligne le 26 août 2011, consulté le 01 mai 2019. URL : http://journals.openedition.org/narratologie/6332; DOI : 10.4000/narratologie.6332

Ce document a été généré automatiquement le 1 mai 2019.

Article L.111-1 du Code de la propriété intellectuelle. 


\title{
Voix-off et film-fable : le cinéma d'éducation populaire à l'épreuve du parlant
}

\author{
Pascal Laborderie
}

\section{Introduction}

1 Le film-fable présente un système d'énonciation qui se dédouble en un récit et un discours, de telle sorte que le spectateur construit à la fois un énonciateur fictif et un énonciateur réel. Or, cette hétérogénéité énonciative du film-fable correspond aux deux grandes fonctions de la voix-off: raconter et tenir un discours. C'est pourquoi l'observation de l'émergence du parlant dans les films-fables semble particulièrement intéressante en ce qui concerne l'étude des caractéristiques médiatiques, narratologiques et idéologiques de la voix-off. Ces premières utilisations de la voix-off peuvent être observées dans les films-fables programmés par les réseaux d'éducation populaire laïque dans les années 1930.

De quels réseaux et de quels films s'agit-il ? Nous pensons principalement au dispositif du « cinéma scolaire et éducateur » institué par les offices régionaux du cinéma éducateur ainsi que par les divers ministères, notamment l'Instruction publique et l'Agriculture. Tandis que les séances scolaires et périscolaires (le " cinéma scolaire ») programment des films d'enseignement qui s'adressent aux élèves, les activités postscolaires et populaires (le " cinéma éducateur ") visent les adolescents et les adultes, qu'il s'agit d'éduquer à la citoyenneté et à l'hygiène sociale. Ce dispositif d'enseignement, d'éducation et de propagande sanitaire et sociale est emblématique des intentions de l'éducation populaire laïque, qui consistent certes à élever le niveau scolaire des mondes ouvriers et paysans, mais aussi à diriger leurs pratiques culturelles, sociales et politiques. Ce mouvement social est inséparable de l'histoire politique de la Troisième République, qui se caractérise par l'hégémonie du parti radical. Ayant réalisé près de trois cents films, Jean Benoit-Lévy 
est certainement le réalisateur le plus important de ce cinéma d'éducation populaire laïque, qui s'inscrit principalement dans les mouvances radicale et socialiste. Ses premiers films parlants, en particulier le Coeur de Paris (1932) et la Maternelle (1933), font l'objet d'un travail spécifique sur la voix-off. Cependant, selon une conception élargie de l'éducation populaire laïque par le cinéma, qui préfigure le Front Populaire, il nous semble possible d'admettre dans la nébuleuse du cinéma éducateur laïc les productions beaucoup moins nombreuses du cinéma militant communiste, en particulier Borinage (Joris Ivens et Henri Storck, 1933) et la Vie est à Nous (collectif CGTU, 1936).

Nous proposons de considérer les modalités et les enjeux de l'apparition de la voix-off dans les films-fables programmés par ces réseaux d'éducation populaire laïque. Tout d'abord, nous envisageons la manière dont s'est opéré le transfert médiatique des genres oraux qui encadraient les séances du cinéma éducateur muet, la conférence et le débat avec le public. À cette occasion, nous remettons en question la valeur démiurgique de la voix-off qui, dans le contexte du cinéma éducateur, rappelle la voix du maître d'école plutôt que celle de Dieu. Aussi, nous distinguons deux catégories de films-fables : le filmconférence dans lequel le récit sert à introduire un exposé et le film-parabole où le récit permet d'illustrer deux comportements opposés. Ensuite, nous étudions pourquoi et comment le film-parabole bannit la voix-off non diégétique et préfère mobiliser la voixoff diégétisée. Dans une perspective narratologique, ce régime intermédiaire se conforme à l'hétérogénéité énonciative du film-parabole. Enfin, d'un point de vue idéologique, nous envisageons les raisons qui ont conduit à la mise à l'index de la voix-off non diégétique. Ce phénomène participe d'un mouvement de contestation de la parole des élites, qui émerge dans le cinéma populaire des années 1930. Il est observable aussi bien dans le cinéma de Jean Benoit-Lévy que dans un film marquant du Front populaire : la Vie est à nous.

\section{La voix du maître d'école}

4 Si la parole dans les films de fiction du cinéma muet a déjà fait l'objet d'études (par exemple sur les intertitres ou sur la place du bonimenteur), sa place dans les films éducatifs du cinéma muet est en revanche peu connue. Pourtant, les séances du cinéma éducateur étaient commentées par l'instituteur ou par les docteurs des services d'hygiène et suivies d'une conférence ou d'un débat avec le public. Elles proposaient un programme de courts métrages qui préservait un équilibre entre les visées éducatives des instituteurs et les goûts plus récréatifs du public d'adultes. Au cours des années 1920, ce programme tendit à disparaitre au profit d'un film unique qui réunissait tous les critères d'un bon programme de cinéma éducateur. Aussi, avant même l'émergence du parlant, le cinéma éducatif muet avait réussi à intégrer à sa manière l'organisation discursive des genres oraux qui encadraient traditionnellement ces programmes de courts métrages: la conférence et le débat. Dans cette perspective, Jean Benoit-Lévy a réalisé deux genres de films-fables qui articulent un récit et un contenu éducatif : le film-conférence dans lequel le récit sert à introduire un exposé (par exemple, la Future maman, 1924) et le filmparabole où le récit illustre deux comportements opposés (par exemple, Âmes d'enfants, 1928).

5 D'une manière générale, l'intégration du cinéma parlant dans les dispositifs d'éducation populaire est tardive par rapport à sa généralisation dans le cinéma commercial. $\mathrm{Ce}$ retard est lié à des obstacles d'ordre économique et idéologique. Tandis que les 
éducateurs souhaitent s'équiper en parlant pour attirer le public d'adultes, les pédagogues sont plus réservés à l'égard du commentaire. Cette réticence est notamment perceptible dans les écrits d'Eugène Reboul, le directeur de l'office de Saint-Étienne. Contrairement à Gustave Cauvin, le directeur de l'office de Lyon, qui développe considérablement le cinéma éducateur parlant, son homologue stéphanois privilégie le cinéma scolaire muet. Plusieurs raisons expliquent cette réserve à l'égard du parlant. Tout d'abord, avant 1936, il n'existe aucun système financier pour sonoriser les écoles et renouveler le fonds cinématographique. Par ailleurs, il semble improbable de trouver suffisamment de films éducatifs parlants pour approvisionner chaque usager en programmes variés à raison de sept à huit séances par semaine. Enfin, Eugène Reboul refuse le cinéma parlant pour des raisons pédagogiques : «Non, dans la classe, rien ne peut remplacer la parole du maître. Celui-ci, s'adaptant à son milieu, voit sur la physionomie de ses élèves s'il est compris et peut continuer, ou s'il doit s'arrêter et reprendre son exposé sous une autre forme ».

6 Nous remarquons que l'objection d'Eugène Reboul contre l'utilisation du parlant dans les séances scolaires n'est pas liée à la crainte d'une remise en cause de l'autorité du maitre, mais participe plutôt d'une volonté d'adapter la séance à la diversité des situations pédagogiques. En fait, les instituteurs doivent composer avec des films d'une mauvaise qualité pédagogique, qu'ils soient muets ou parlants. C'est pourquoi ils finissent par réaliser leurs propres films. En 1932, Jean Brérault réalise ainsi son premier film d'enseignement parlant (En Bretagne, 1933). Par ailleurs, les animateurs des Offices réalisent eux-mêmes des films parlants, notamment Arthur Gautier, le directeur de l'office de Nîmes, et Louis Colin, le directeur de l'office de Nancy. Aussi, contrairement à l'office de Saint-Étienne, l'office de Nancy s'engage en faveur du parlant et produit le Massif Vosgien (Louis Colin et André Dolmaire, 1936), En se donnant la main (Louis Colin et André Dolmaire, 1937) et le Jura (Louis Colin et André Dolmaire, 1939 et 1946). Tous ces films se caractérisent par l'utilisation d'une voix-off non diégétique qui, dans le contexte du cinéma scolaire et éducateur, rappelle bien évidemment le commentaire du maitre d'école. Afin de prendre en considération cet héritage, nous proposons en quelque sorte de laïciser la voix-off traditionnellement considérée comme la voix de Dieu (« the Voiceof-God commentary »).

\section{La voix-off dans le système énonciatif du film- parabole}

Malgré certaines réticences de la part des pédagogues, les films d'enseignement finissent ainsi par adopter la voix-off qui se substitue aux intertitres à valeur de commentaire. Les films-paraboles, plus proches de la fiction, évitent quant à eux de mobiliser la voix-off non diégétique et développent la musique, les sons d'ambiance et les dialogues (par exemple dans la Maternelle, Jean Benoit-Lévy et Marie Epstein, 1933). L'utilisation de la voix-off bouleverse en effet l'économie énonciative du film-parabole et bloque en particulier l'effet-fiction. L'étude comparée de deux versions de Borinage, l'une muette (Joris Ivens et Henri Storck, 1933), l'autre parlante (1963) permet ainsi d'observer ce que le parlant fait au film-parabole et de montrer que l'utilisation de la voix-off contribue à changer les modalités de lecture du film. 
8 Le film commenté de 1963 reprend la bande-image de 1933. Les intertitres ont été escamotés et leur contenu réintégré par la voix-off. Ce commentaire redistribue mot pour mot l'ensemble des mentions écrites à l'exception du titre et d'un seul intertitre. Dans la séquence où une famille de mineur est expulsée et trouve assistance dans un autre foyer, la famille d'accueil est d'abord désignée par un intertitre comme une famille faisant preuve de solidarité (ce qui alimente le discours procommuniste), tandis que dans le film de 1963, le commentaire explique qu'il s'agit tout simplement de la belle-famille du mineur. Aussi, la comparaison des deux films est-elle particulièrement intéressante concernant l'étude de la voix-off et constitue même un cas d'école dans la mesure où celle-ci constitue la seule variable intrinsèque au film. Tandis que Borinage mobilise largement la lecture parabolisante en construisant systématiquement un discours procommuniste à partir de séquences qui racontent la vie des mineurs, Misère au Borinage favorise plutôt une lecture documentarisante. Les intertitres, parce qu'ils se situent en amont ou en aval des séquences, aménagent une économie énonciative plus souple que celle instaurée par la voix-off. La relation du commentaire aux images conduit à une redondance et à une prédominance de la voix-off qui brident les potentialités fictionnelles du film de 1933. En outre, le ton du commentaire, quoique d'une manière générale fort neutre, laisse percer à deux reprises une pointe d'ironie. Cette forme d'adresse au spectateur participe du même processus qui bloque la construction d'un énonciateur fictif au profit d'un énonciateur réel. De fait, tandis que Borinage (1933) fonctionne comme un film-parabole, la voix-off contribue largement à faire basculer Misère au Borinage (1963) du côté d'un mode de lecture documentarisant.

9 Ainsi, la voix-off non diégétique semble incompatible avec le régime énonciatif du filmparabole. Cependant le film-parabole peut chercher à s'accommoder de la voix-off en la diégétisant. Cette tentative est notamment perceptible dans les premiers films parlants de Jean Benoit-Lévy. En 1930 et 1931, il réalise respectivement Jimmy Bruiteur et le Cour de Paris, où il entreprend d'expérimenter la relation image-son dans toutes ses dimensions. Située dans le prolongement de cette exploration systématique, la Maternelle est pourtant marquée dans son générique d'ouverture par un retour aux mentions écrites. Ce film sonore est ainsi dans un premier temps privé de parole. L'utilisation de la parole auraitelle été différée afin de préserver un équilibre énonciatif fragile? Concernant l'articulation des deux micro-systèmes d'énonciation mise en œuvre dans la «fabulisation », Roger Odin souligne qu'il s'agit d'une question de " dosage » et qu'il est du reste possible de « repérer toute une série d'intermédiaires entre le film-fable [...] et le film de fiction ». Dans cette perspective, l'introduction de la parole dans la Maternelle nous semble faire l'objet d'un traitement spécifique. Au début du film, un couple danse dans une soirée mondaine. La musique est ainsi d'emblée diégétisée. Puis les deux amants s'écartent de la piste de danse. La jeune femme s'assoit. Un plan rapproché la montre arborer un sourire radieux. Elle est vêtue d'une robe blanche. À l'arrière plan, un bouquet de fleurs blanches semble couronner sa tête. Sur cette figure annonciatrice d'un mariage, la première parole du film est enfin prononcée à voix basse par une voix masculine : «toujours ». À la fin de la séquence, l'homme passe une bague de fiançailles à l'annulaire gauche de la jeune femme et lui baise la main. Naturellement, il nous semble intéressant d'analyser le régime de cette voix qui prononce le mot « toujours».

En raison de son caractère acousmatique, il est possible de la qualifier soit de voix horschamp, soit de voix-off. Tout d'abord, la masculinité de la voix et la logique de l'action laissent penser qu'il s'agit d'une parole prononcée par l'amant de la jeune femme. 
Cependant elle apparaît sur l'image sans qu'aucun plan situé avant ou après ne vienne confirmer par la voix-in qu'il s'agit bien d'une voix hors-champ. Outre l'absence de voixin, un autre élément inscrit cette parole dans le régime de la voix-off : « toujours » est un mot qui survient isolément. S'il s'agit d'un morceau de dialogue volontairement tronqué, ce travail de coupe est suffisamment marqué pour sortir le spectateur de la fiction. Mais il est tout autant possible de considérer que « toujours » constitue un résumé des propos de l'amant qui seraient rapportés avec une certaine liberté. Dans les deux hypothèses, l'isolement de cette parole contribue à un processus de discursivisation de la voix. En définitive, située sur la frange dans laquelle hors-champ et off s'interpénètrent, cette voix correspond à l'hétérogénéité énonciative du film-parabole dans le sens où il construit à la fois un récit et un discours.

\section{Dissensions vocales dans le cinéma de Jean Benoit- Lévy}

11 La voix-off semble peu compatible avec le système énonciatif du film-parabole. Elle est par ailleurs difficilement admise par le cinéma d'éducation populaire pour des raisons non plus narratologiques, mais idéologiques. Par exemple, le cinéma de Jean Benoit-Lévy, s'il participe avant tout d'une propagande en hygiène sociale de type sociologique, supporte également un discours politique d'obédiences radicale et socialiste. Aussi, les films de Jean Benoit-Lévy expriment-ils les tensions sociales qui apparaissent dans l'entre-deux-guerres. Dans cette perspective, la voix-off et la voix des élites sont tour à tour contestées dans le Cour de Paris et la Maternelle.

La première séquence du Coeur de Paris est un pastiche du documentaire touristique. Dans un premier temps, le film passe en revue tous les monuments-phares de Paris qui sont désignés par un commentaire off non diégétique : «Voici l'Opéra, la Madeleine, la Place de la Concorde, la Tour Eiffel, le Moulin de la Galette, le Sacré Cœur ». Puis la vue d'un autocar touristique vient réinscrire cette visite dans l'univers du film. Des titis parisiens, parmi lesquels on reconnait Jimmy Gaillard, regardent le bus s'avancer. Debout sur la plate-forme panoramique du bus, un guide tient un porte-voix et s'exprime en plusieurs langues. Le commentaire off est ainsi diégétisé après coup. Le guide insulte les enfants en les traitant d'apaches. Suit une rixe où les enfants finissent par faire taire le guide qui, littéralement, a la gueule cassée. Du reste, Jimmy Gaillard s'exclame avec un accent parigot digne de celui de Carette : " pauvre type, on lui a démoli son garde-manger ! ». Ce travail de typage sur le registre de langue, le ton et l'accent permet bien évidemment d'opposer le parler populaire des enfants à la parole du guide. Jimmy Gaillard s'empare du porte-voix du guide irrémédiablement réduit au silence et entreprend de le remplacer à sa manière : «Miss, Fraulein, señorita, pour vous remercier, j'vais vous montrer Paname! Et nous allons voir la Bastoche, le Boul'Mich, le Louvre, le Bateau-Lavoir et toutes les boîtes de nuit, en avant!». Ce basculement d'une voix off non diégétique qui commente sur un ton neutre les monuments parisiens vers une voix-in aux accents populaires, cette revendication de la parole par le peuple, nous semble représentative du cinéma de Jean Benoit-Lévy tel qu'il préfigure le Front populaire.

13 Cette affirmation du parler populaire est aussi observable dans la Maternelle. Une séquence y raconte la visite d'un recteur dans une classe. Ce recteur a fait parvenir à l'école un lapin à l'aide duquel il souhaite récapituler une expérience pédagogique. La 
découverte du rongeur attendrit naturellement les petits enfants. Puis le recteur, qui tient la classe en lieu et place du maître, s'adresse aux enfants sur un ton professoral : « Je vais demander à Madame la Directrice qu'on le fasse porter à la cuisine afin que la cantinière le prépare pour le déjeuner ». Cette proposition énoncée en voix hors-champ laisse découvrir la réaction d'une petite fille qui s'exclame indignée : « Non, non, non, faut pas le tuer, le petit lapin!». Finalement, les enfants se révoltent et s'écrient en chœur avec un accent parisien : « Oh, quel salop salaud! Quel salop salaud! ».

Ce cinéma d'éducation populaire, tel qu'il émerge entre le Cartel des Gauches et le Front populaire, donne ainsi la parole au peuple tout en critiquant la parole des élites et ses multiples avatars, en l'occurrence la parole du guide ou encore celle du recteur. Plus exactement, il aménage un espace de mixité sociale, où les diverses paroles peuvent cohabiter. Cette mise en scène de la parole évoque les tensions sociales tout en les euphémisant au profit d'un discours modéré consensuel.

\section{La voix-in est à nous !}

Moins consensuel que la Maternelle, la Vie est à nous évoque de manière plus tranchée les dissensions vocales entre la parole du maitre (en voix-off) et la parole du peuple (en voixin). Jean Dasté y joue le rôle d'un instituteur qui fait un cours de géographie économique. Plus exactement, au début du film, des plans à valeur documentaire sont commentés par une voix dont il est tout d'abord impossible d'identifier la source. Le film commence comme une bande d'actualité ou un film d'enseignement géographique commenté par une voix-off non diégétique. Puis un plan de l'instituteur poursuivant son cours au tableau vient réinscrire cette voix-off non diégétique dans l'univers du film et en l'occurrence dans le cadre d'une leçon. Par la suite, la séquence alterne la leçon du maître (en voix-in) avec des plans documentaires commentés en voix-off diégétisée. Dans une perspective médiatique, cette ouverture nous semble emblématique du transfert de la parole de l'instituteur dans le commentaire, tel qu'il s'est effectué dans les films éducatifs au milieu des années 1930.

D'un point de vue narratologique, cette ouverture constitue un bel exemple de diégétisation du commentaire off. Le passage de la voix-off non diégétique à la voix-in aménage un effet de surprise. Nous notons que cette transition s'opère au moment d'une pause du commentaire. De plus, l'instituteur apparaît pour la première fois dos à la caméra et tourné vers un tableau. Cependant une interrogation peut aussi bien porter sur la valeur des images documentaires que sur le régime de la voix. En effet, si le tableau peut évoquer de manière symbolique un écran de cinéma, il ne s'agit pas pour autant d'une leçon au moyen d'une projection cinématographique. Aussi, les images documentaires constituent-elles soit une illustration du commentaire du maître entièrement prises en charge par un énonciateur réel, soit les images mentales des personnages assumées par un énonciateur fictif. C'est pourquoi la voix du maître est assimilable à une voix-off diégétisée plutôt qu'à une voix hors-champ.

Dans le courant de la séquence, cette voix adopte alternativement les régimes du in et du hors-champ. À la fin de la séance, elle connaît un dernier infléchissement. Après la sortie des enfants, le maître qui demeure seul à l'écran soupire: "pauvres gosses». Ce monologue dit en voix-in a une valeur de commentaire. Il pointe un écart entre le discours officiel sur la richesse de la France tenu dans le cadre de la leçon et la situation sociale des enfants. Cette discursivisation de la voix-in répond au mouvement de 
diégétisation de la voix-off. Elle obéit en effet à cette contrainte narratologique du filmparabole qui consiste à préserver un équilibre entre récit et discours.

Enfin, selon ses visées idéologiques, le film met en relation la parole du maître et celle des élèves. Par la même occasion, il évoque indirectement les deux genres oraux qui accompagnent habituellement les séances du cinéma scolaire et éducateur, c'est-à-dire l'exposé de l'instituteur et le débat avec le public. Le dispositif du cinéma scolaire muet inscrivait la leçon au moyen du cinéma dans le cadre d'un cours dialogué. Dans la Vie est à nous, cette possibilité d'interaction est représentée à la sortie de la classe, où un élève vient poser une question au maître au sujet de la production de volaille. La séquence suivante s'inspire quant à elle du dispositif du cinéma éducateur dans la mesure où la leçon suscite dans la rue un vif débat entre les enfants. Le diagnostic du maitre quant à la situation économique florissante de la France est confronté à la situation sociale désastreuse des enfants. La dénonciation de ce paradoxe est un élément bien connu de la propagande communiste. Dans le contexte du film, elle annonce la stigmatisation des plus grandes fortunes de France, les fameuses deux cents familles : «La France n'est pas aux Français, car elle est à deux cents familles. La France n'est pas aux Français, car elle est à ceux qui la pillent ». Ce discours populiste est lui-même perceptible dans l'affirmation du parler populaire. À l'instar des enfants du Cœur de Paris, les enfants de la Vie est à nous ont un accent parigot bien prononcé qui contraste avec la posture professoral du discours officiel incarné par la voix-off, telle qu'elle apparaissait au début du film.

\section{Conclusion : Vox populi, vox dei}

En définitive, cette étude nous a permis d'étudier la voix-off telle qu'elle a émergé dans les films-paraboles du cinéma d'éducation populaire laïque. Traditionnellement assimilée à la voix de Dieu, cette voix nous semble plutôt déterminée d'un point de vue pragmatique par le dispositif du cinéma scolaire et éducateur. C'est pourquoi nous proposons de séculariser la voix-off non diégétique et d'y voir un héritage du commentaire du maître d'école qui accompagnait le cinéma scolaire et éducateur muet. Pour autant, cette voix du maitre pose problème aussi bien à l'idéologie du film d'éducation populaire qu'au régime énonciatif du film-parabole qui en constitue l'expression formelle. Dans une perspective narratologique, le film-parabole préserve un équilibre entre deux micro-systèmes énonciatifs qui construisent à la fois un énonciateur réel et un énonciateur fictif. C'est pourquoi il cherche à aménager un régime de voix intermédiaire (la voix-off diégétisée). En ce qui concerne son idéologie, le film d'éducation populaire conteste la voix-off non diégétique en tant qu'elle rappelle la parole du maître et plus généralement celle des élites. La séquence d'ouverture de la Vie est à nous est symptomatique de ce triple mouvement médiatique, narratologique et idéologique, qui assimile la voix-off à la parole du maitre pour mieux mettre en valeur la parole du peuple. 


\section{RÉSUMÉS}

Comment l'étude de la généralisation du parlant dans les films-fables du cinéma éducateur permet-elle de comprendre la participation de la voix-off dans la non-fiction ? L'ambivalence de l'économie énonciative des films-fables correspond aux deux grandes fonctions de la voix-off: raconter et supporter un discours. Aussi, l'observation de l'émergence du parlant dans ces films permet-elle d'étudier le statut de la voix-off dans les films de non-fiction. C'est pourquoi nous proposons de considérer les modalités et les enjeux de l'apparition de la voix-off dans les filmsfables programmés par les réseaux d'éducation populaire laïque dans les années 1930. Dans un premier temps, nous envisageons la manière dont s'est opéré le transfert médiatique des genres oraux qui encadraient les séances du cinéma éducateur muet, la conférence et le débat avec le public. À cette occasion, nous remettons en question la valeur démiurgique de la voix-off qui dans le contexte du cinéma éducateur rappelle la voix du maître d'école plutôt que celle de Dieu. Aussi, nous distinguons deux catégories de films-fables : le film-conférence dans lequel le récit sert à introduire un exposé et le film-parabole où le récit permet d'illustrer deux comportements opposés. Dans un second temps, nous étudions pourquoi et comment le film-parabole mobilise un régime de voix intermédiaire : la voix-off diégétisée. Dans une perspective narratologique, ce régime se conforme à l'hétérogénéité énonciative du film-parabole. D'un point de vue idéologique, l'utilisation de cette voix-off diégétisée participe de ce mouvement de contestation de la parole des élites, qui émerge dans le cinéma populaire des années 1930.

\section{INDEX}

Index géographique : France

Mots-clés : cinéma éducateur, éducation populaire, enseignement, Front Populaire, non-fiction, voix-off, voix-over

Index chronologique : 1918-1939

\section{AUTEUR}

\section{PASCAL LABORDERIE}

Pascal Laborderie a soutenu une thèse intitulée Le film-parabole dans les Offices du " cinéma

éducateur ». Histoire d'un cinéma de propagande et étude d'un genre cinématographique (Paris 3, 2009). Il est PRAG en Sciences de l'Information et de la Communication à l'IUT de Troyes, Université de Reims Champagne-Ardenne. Il est associé à l'Institut de recherche sur le cinéma et l'audiovisuel. Dernières contributions : « Le Voile sacré (Jean Benoit-Lévy, 1926), un film d'éducation populaire dans le réseau du cinéma éducateur laïque " (L’image dans l'histoire de la formation des adultes, dir. Françoise F. Laot, Paris, L'Harmattan, 2010, pp. 31-48) et « Les Offices du cinéma éducateur et l'émergence du parlant : l'exemple de l'Office de Nancy » (1895, à paraître fin 2011). 